\title{
LE PROBLÈME DE LA REPRÉSENTATION ET SA SOLUTION Dans la linguistique de Gustave Guillaume, LeCTeUr de SAUSSURE
}

\author{
Philippe Monneret \\ Université Paris-Sorbonne / EA 4509 STIH, Paris, France
}

\section{Résumé}

La centralité du concept de représentation chez Gustave Guillaume ne provient pas de la nature de l'objet de son étude, quel que soit le nom que l'on choisisse de lui donner: la langue, les langues ou le langage. Car une linguistique pourrait fort bien s'en passer, ou lui conférer un statut accessoire en préférant un autre concept directeur - la sémiose par exemple dans le cas de la linguistique saussurienne. Il n'est donc guère étonnant qu'on puisse finalement se demander à quel genre de problème répond la présence centrale du concept de représentation dans la linguistique de Gustave Guillaume. Si «promouvoir le langage à l'existence, c'est le promouvoir à la représentation - ce sans quoi rien n'existe pour l'esprit», ce n'est pas en raison de la nature du langage que le linguiste recourt au concept de représentation mais parce qu'il existe une certaine façon d'être un linguiste qui consiste à poser le langage comme un objet pour l'esprit, attitude qui se distingue, parmi bien d'autres solutions, de celle consistant par exemple à le voir comme un objet ou un "paramètre» de la vie sociale.

\section{Mots-clés}

représentation, système, Gustave Guillaume, psychomécanique du langage, arbitraire du signe, analogie

\begin{abstract}
The centrality of the concept of representation in the work of Gustave Guillaume does not derive from the nature of the object of his study, language or languages, because a linguistic theory could very well do without it, or give it an accessory status by preferring another fundamental concept - for example, in the case of Saussurian linguistics, semiosis. It is therefore hardly surprising that one can ultimately wonder what kind of problem it is to which the central presence of the concept of representation in the linguistics of Gustave Guillaume is meant as a response. If "to promote language to existence is to promote it to representation - without which nothing exists for the mind", it is not because of the nature of language that the linguist resorts to the concept of representation, but because there is a certain way of being a linguist, which consists in posing language as an object for the mind, an attitude which is distinct, among many other solutions, from that consisting, for example, in seeing it as an object or a "parameter" of social life.
\end{abstract}

\section{Keywords}

representation, system, Gustave Guillaume, psychomechanics of language, arbitrariness of the sign, analogy

En quoi la lecture de Saussure par Guillaume peut-elle éclairer certaines potentialités du texte saussurien? La question sera examinée ici à partir du concept de représentation. Je commencerai par une brève comparaison fondée sur l'examen de la place qu'occupe la notion ou le concept de représentation chez Guillaume et chez Saussure pour m'attacher ensuite à une présentation des différents emplois de 
la notion de représentation chez Guillaume. Cela permettra de montrer que ce qui est en jeu dans cette notion de représentation, chez Guillaume, repose sur la notion de «système de la langue », que l'on pourra comparer avec l'utilisation qu'en fait Saussure.

Quelle est la place de la notion de représentation chez Saussure et chez Guillaume? Chez Saussure, lorsqu'on consulte l'index de l'édition française du Cours de linguistique générale, l'entrée «représentation» est absente. Elle n'apparaît pas non plus dans l'index des Écrits de linguistique générale. Les seules occurrences remarquables sont liées à l'idée de «représentation de la langue par l'écriture». D'une manière générale, l'emploi par Saussure du terme représentation vise classiquement à désigner le rapport entre les entités formelles et les entités sémantiques ou idéelles. Par exemple :

Toute l'étude d'une langue comme système c'est-à-dire d'une morphologie revient, comme on voudra, à l'étude de l'emploi des formes, ou à celle de la représentation des idées. Ce qui est faux, c'est de penser qu'il y ait quelque part des formes (existant par elles-mêmes hors de leur emploi) ou quelque part des idées (existant par elles-mêmes hors de leur représentation). (Saussure 2002, p. 31)

La situation est tout à fait différente chez Guillaume. En effet, la consultation de l'index de la base de données Gustave Guillaume ${ }^{1}$, qui n'est pas exhaustive mais contient tout de même 27 volumes numérisés, permet de constater immédiatement que la notion de représentation joue dans l'œuvre un rôle considérable (83 occurrences) :

représenté architectural / représenté construit / représenté institué / représenté oral / représenté saisi / représenté scriptural / représenté spatial / représenté systématique / représenté / représentabilité / représentable / représentation abstrait(e) / représentation architectural(e) du temps / représentation architectural(e) spatialisé(e) / représentation arithmétisé(e) / représentation ascendant(e) / représentation ascendant(e) du temps / représentation bi-linéaire / représentation d'objet / représentation d'un procès / représentation d'univers / représentation de l'étendue de la pensée / représentation de la personne / représentation de langue / représentation descendant(e) du temps / représentation dicible / représentation dimorphe du temps / représentation distinct(e) / représentation du hors-moi / représentation du nombre / représentation du temps / représentation en langue / représentation essentiel(e) / représentation exo-sémantique / représentation explicite / représentation figuratif(ve) / représentation général(e) du pensable / représentation généralisé(e) / représentation graphique / représentation horizontal(e) / représentation horizontal(e) du temps / représentation idéel(le) / représentation irrationnel(le) / représentation large du temps / représentation lexical(e) / représentation linéaire du temps / représentation linéaire extensif(ve) du passé / représentation linguistique / représentation linguistique du temps / 
représentation matériel(le) / représentation mental(e) / représentation morphologique / représentation morphologique du temps / représentation notionnel(le) / représentation partitif(ve) / représentation physique / représentation pictural(e) d'objet / représentation plan(e) du temps / représentation positif(ve) du temps / représentation préalable / représentation premier(ère) / représentation second(e) / représentation singularisé(e) / représentation spatial(e) / représentation spatialisé(e) / représentation spatialisé(e) du temps / représentation sublimé (e) / représentation substantiel(e) / représentation sub-verbal(e) / représentation systématique / représentation systématisé(e) / représentation systématisé(e) du temps / représentation systématisé(e) intégral(e) / représentation / représentation temporel(le) / représentation trimorphe du temps / représentation uni-linéaire / représentation universalisant / représentation universel(le) / représentation vectoriel(le)/ représentation verbal(e) / représentation vertical(e $)^{2}$

En dépit du déséquilibre quantitatif des corpus comparés, il s'agit là d'un indice montrant qu'il existe probablement, entre Saussure et Guillaume, une importante différence de traitement du concept de représentation.

\section{LE CONCEPT DE REPRÉSENTATION DANS LA LinguistiQue de GustaVE} Guillaume

\subsection{Langue, discours et représentation}

Je commencerai par ce qui est sans doute le plus connu : la représentation comme phase de la transition entre langue et discours (fig. 1).

Le fait de langue est constitué d'actes de représentation qui aboutissent à des représentations instituées (distinction entre le processus de représentation et son résultat: une représentation). Bien évidemment, ce qu'il y a de spécifiquement guillaumien dans cette distinction devenue usuelle entre langue et discours (terme délibérément choisi par Guillaume contre le terme saussurien de parole), c'est le fait qu'elle est conçue non pas comme une dichotomie mais en termes temporels, comme un processus décomposé en deux phases. Plus précisément, contrairement à l'idée - apparemment partagée par Saussure - selon laquelle la représentation se situerait seulement du côté du signifié, de la signification ou des idées, la conception guillaumienne de la représentation implique qu'elle intervient aussi au plan du signifiant (au plan de la phonologie), comme l'indique la figure 2 cidessous.

Le plan de la phonétique ou de la réalisation phonétique des phonèmes se situe au niveau du discours, donc sur le même plan que les actes d'expression. Cette distinction entre représentation et expression, entre langue et discours, est sousjacente également à la distinction entre signifié de puissance et signifié d'effet, l'une des particularités de la terminologie de Guillaume, qui distingue deux plans du

2 Ajoutons que chacune de ces entrées d'entrées d'index renvoie à de nombreuses occurrences dans les textes (jusqu'à plusieurs dizaines). 


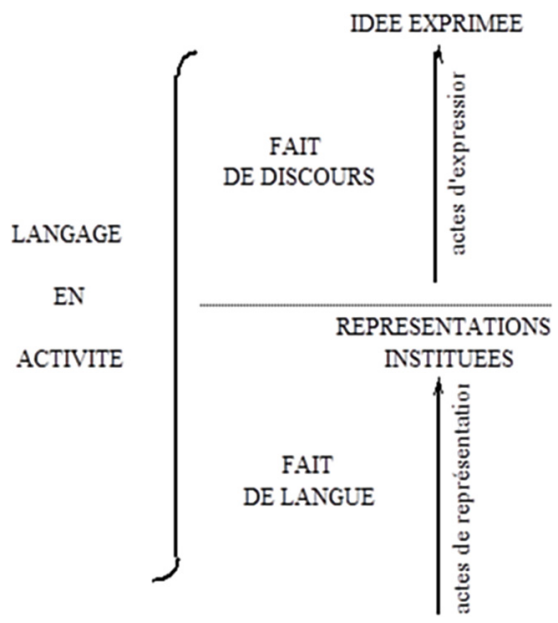

Figure 1 : (Guillaume 1949, p. 17).

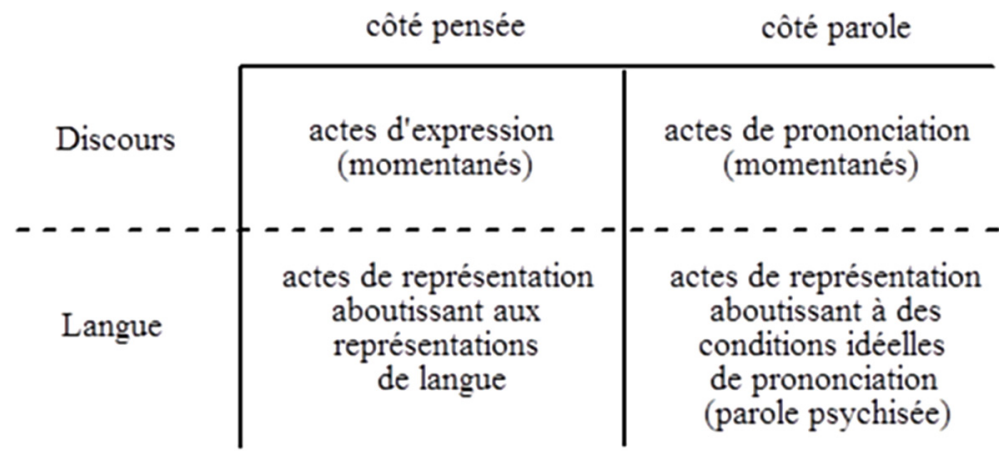

Figure 2 : (Guillaume 1949, p. 71).

signifié : un plan du signifié de puissance, qui se situe au niveau de la langue et qui procède d'une recherche d'unification sémantique dans la diversité des emplois discursifs, celle-ci ayant pour dénomination signifié d'effet. Les signifiés d'effet dérivent des signifiés de puissance par un processus de saisie sur un mouvement, puisque les signifiés chez Guillaume sont toujours des mouvements de pensée ou des mouvements (processus) de représentation. Ce type d'analyse peut être complété par un troisième niveau, celui des effets de sens, comme le montre la figure 3 ci-dessous.

À titre d'exemple, le signifié de puissance de l'article défini est un mouvement de particularisation, les signifiés d'effet du même article défini sont principalement ses emplois génériques (résultant d'une saisie située au début du mouvement de particularisation, donc à un moment qui demeure de grande généralité) et ses emplois spécifiques (résultant d'une saisie située à la fin du mouvement de 


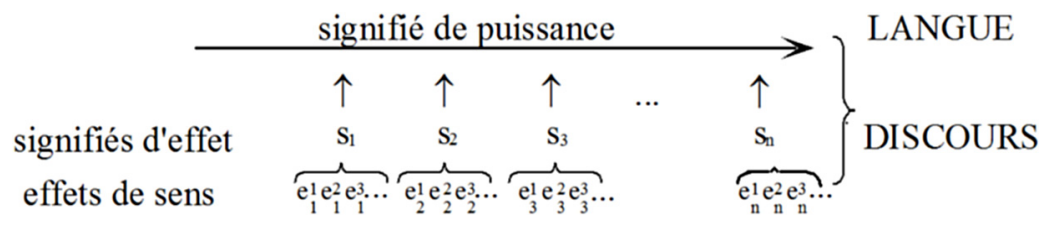

Figure 3 : Position des effets de sens dans la transition langue-discours (Monneret 2003, p. 19).

particularisation, donc à un moment de particularité maximale). Enfin, toujours dans le cas de l'article, les effets de sens correspondent aux emplois possibles des articles spécifiques ou génériques (par exemple, dans le cas des valeurs spécifiques, l'emploi anaphorique ou l'emploi déictique). Ajoutons que, pour Guillaume, le rapport entre signifiant et signifié est également un rapport de représentation: le signifiant représente le signifié.

La citation suivante montre bien l'importance pour Guillaume de ce rapport entre représentation et expression:

Le fait humain, c'est le rapport représentation / expression. Ce fait est, en matière linguistique, de tous les temps, de tous les âges, de toutes les sociétés et socialités. Ce qui veut dire que partout, toujours, le langage parlé se trouve en présence du problème d'un équilibre représentation / expression. Il s'agit, par le moyen d'actes de représentation, de faire la langue et, par le moyen d'actes d'expression, de faire le discours.

La relation obligée - s'il doit y avoir langage parlé humain (le langage parlé de l'homme) - entre représentation et expression est une relation humaine, tandis que la réalisation de cette relation, selon une certaine proportion des conditions obligées intervenantes (représenter/exprimer), est un fait social (Leçon du 23 novembre 1951 (Guillaume 1986, p. 171)).

Par opposition à ce fait humain qu'est le rapport expression / représentation, est donc définie comme fait social la réalisation de cette relation, qui sera nécessairement plurielle, comme le sont les signifiés d'effet ou les effets de sens par opposition aux signifiés de puissance. Le concept de représentation joue bien chez Guillaume sur le plan humain, autrement dit cognitif. On se doute bien sûr que si le concept de représentation est moins important chez Saussure qu'il ne l'est chez Guillaume, c'est parce que, chez le premier, c'est le fait social qui joue le rôle majeur.

\subsection{Les systèmes de la langue comme représentations}

Le deuxième type d'emploi que j'examinerai du terme représentation est le cas de la représentation des systèmes, et je l'illustrerai en présentant le cas particulier de la représentation du temps, l'un des apports majeurs de la linguistique guillaumienne, et qui permet de constater que Guillaume accorde une importance de premier plan à la différence entre la représentation comme résultat et la représentation comme processus. Guillaume, au tout début de Temps et verbe, commence par rappeler que, 
le plus souvent, dans les grammaires du français, trois époques sont distinguées : passé, présent, futur. La critique qu'il propose de ce type de représentation du temps est instructive à l'égard de sa propre conception du terme représentation:

La grammaire traditionnelle, lorsqu'elle traite du temps, ce à quoi elle est tenue au chapitre du verbe, vu que le propre du verbe est d'être sous-tendu de temps, le considère invariablement comme une ligne infinie, recomposée de deux segments dans le prolongement l'un de l'autre, le passé et le futur, que distingue la coupure, insérée entre eux, du présent. Soit figurément:

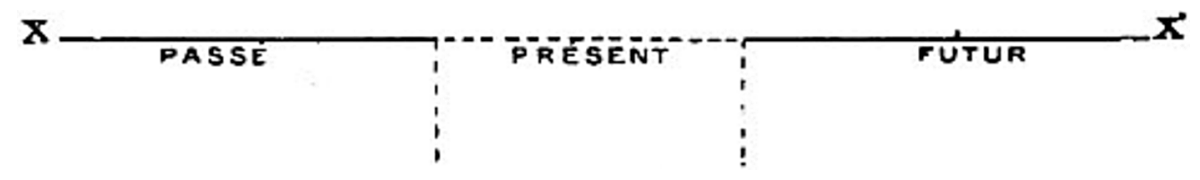

Cette figuration, dont on peut dire qu'elle porte au maximum le panoramisme du temps, est la plus achevée, la plus « réalisée » qu'on puisse concevoir [...] Mais pour le linguiste, et les fins qu'il poursuit, cette image optima du temps est un instrument insuffisant. Son défaut vient précisément de sa "perfection». Ce qu'elle offre au regard, c'est du temps déjà construit en pensée, si l'on peut s'exprimer ainsi, alors que l'analyse demanderait qu'on vît du temps en train de se construire dans la pensée. Il est concevable, en effet, que pour s'introduire profondément à la connaissance d'un objet, cet objet fût-il le temps, point ne suffit de le considérer à l'état achevé, mais qu'il faut de plus, et surtout, se représenter les états par lesquels il a passé avant d'atteindre sa forme d'achèvement. (Guillaume 1929, p. 8)

Ce passage est très représentatif de la démarche générale de Guillaume, qui consiste à reprocher aux grammairiens ou aux comparatistes d'examiner des formes mais de ne pas prendre en considération le fait que ces formes ne sont que des résultats (ou des traces, dans la perspective culiolienne) de processus. La représentation du temps chez Guillaume est un processus de construction de la représentation du temps; les trois époques passée, présente, future ne sont pas un donné de l'expérience humaine, mais résultent d'une opération de construction, qui passe d'une représentation amorphe du temps (modes quasi-nominaux) à une représentation trimorphe (mode indicatif). Cette conception de la représentation du temps est figurée selon diverses schématisations dans l'œuvre de Guillaume, dont je donne ici un exemple, extrait d'une Leçon de mars 1939 (fig. 4), le schéma étant précédé de la présentation suivante:

Les profils distincts que la pensée parvient à prendre de l'opération de pensée engagée sur cet axe, opération de pensée que je nomme chronogénèse, constituent des moments caractéristiques de la réalisation mentale du temps. Dans une langue comme le français, qui a beaucoup et clairement systématisé, ces profils sont exactement au nombre de trois : départ, milieu, arrivée. Ainsi la chronogénèse comporte dans la langue trois vues d'elle-même, initiale, médiane, finale. 


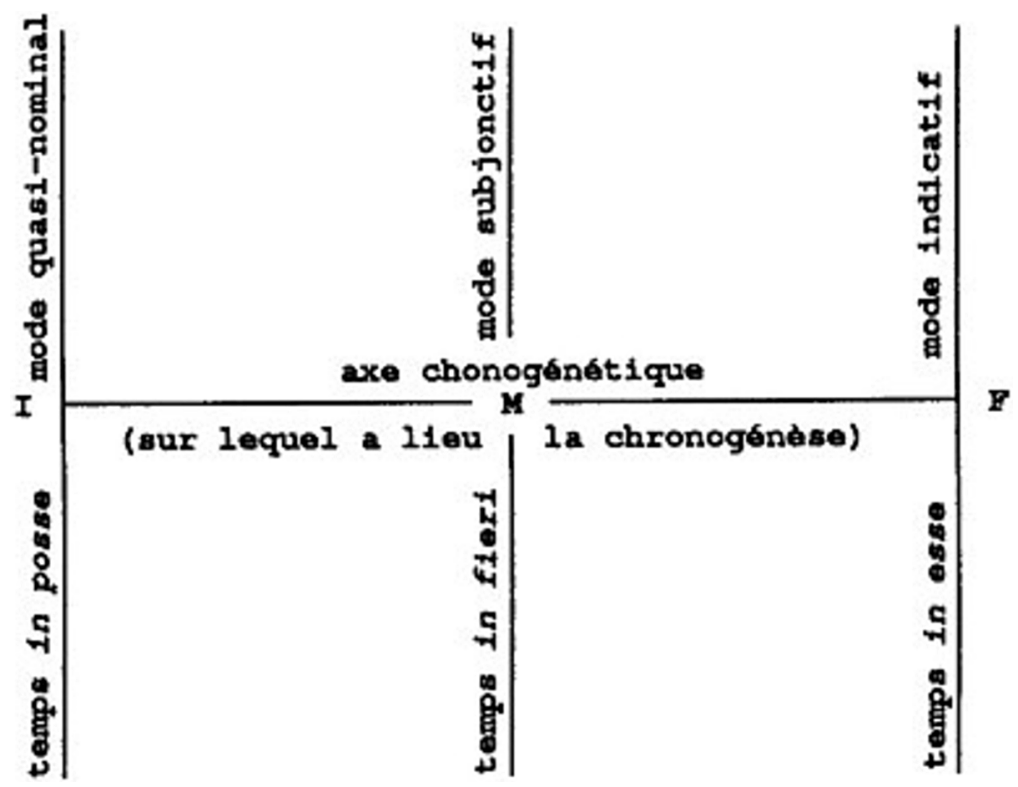

Figure 4 : (Guillaume 1939, p. 165).

Notons au passage que cette représentation n'est pas seulement une construction abstraite réduite à une fonction de description ou d'analyse linguistique. Dans la perspective guillaumienne, une représentation de ce type permet un accès direct, immédiat, à un certain genre de connaissance : « Il suffit d'avoir une vue claire de ce mécanisme fort élégant pour, d'un coup, s'être introduit fort avant à la connaissance de la structure des langues, en ce qui concerne la représentation du temps linguistique »(Guillaume 1945, p. 23).

\subsection{La représentation comme problème de pensée}

Dans son premier livre, Le problème de l'article et sa solution dans la langue française, Guillaume s'intéresse à un changement linguistique particulier: le fait que le français ait développé un article que le substrat latin ignorait. Il s'agit d'une question classique chez les diachroniciens du français mais il est intéressant d'examiner comment Guillaume présente cette question:

Le problème de pensée dont l'article est solution est un problème occulte qui ne nous est pas livré. Il existe au fond de l'esprit et ne peut être restitué qu'indirectement par la comparaison de ce qui a lieu à différentes époques dans plusieurs langues. Chaque système d'article constitue un ensemble d'attitudes intellectuelles que le sujet pensant est capable d'adopter à l'égard du nom. (Guillaume 1919, p. 63)

On retiendra de ces considérations que l'article est suscité originairement par le besoin d'une représentation plus intense, plus originale, que celle qui résulterait du nom seul; qu'il semble ainsi avoir pour cause une certaine insuffisance du 
nom, sensible à l'esprit au moment de l'emploi, et la nécessité corrélative de parer à cette insuffisance par une correction, une retouche. (Guillaume 1919, p. 17-18)

La représentation apparaît ainsi sous l'angle du «besoin», un besoin de représentation qui résulte d'une insuffisance perçue, à une époque donnée de l'histoire d'une langue, dans les représentations disponibles. Le fait de présenter certains facteurs du changement linguistique comme des "problèmes de pensée» est de nouveau une spécificité du guillaumisme: à ma connaissance, la question n'avait jamais auparavant été posée de cette manière. Or ces problèmes de pensée ne sont autres que des problèmes de représentation. Le concept de représentation prend donc ici une dimension collective, sociale. Il n'est plus seulement question d'une représentation au sens d'un processus cognitif individuel mais au sens d'un processus cognitif collectif, donc relevant du plan de la cognition sociale.

\subsection{La représentation comme condition cognitive}

Le dernier type d'emploi que j'examinerai du terme représentation peut être présenté à partir de la «théorie des aires ». Grossièrement, il s'agit d'une théorie typologique qui réduit l'ensemble des langues à trois grandes aires, considérées comme de «états structuraux» du langage humain.

Dans la première aire, un seul espace de construction est possible, celui du mot (qui tient lieu à la fois d'unité de langue et d'unité de discours):

Aire I : la langue et le discours sont « logés » dans un seul espace de construction (le mot): «On trouve dans les langues de l'aire prime plusieurs types d'architectures: type holophrastique (rémanence d'holophrase, p.ex. en basque); type des langues traditionnellement dites «agglutinantes» (p.ex. turc, hongrois, finnois, coréen, etc.); type des langues dites « isolantes» (p. ex. chinois)» (Boone et Joly 1996, p. 42-43)

La seconde aire est celle des langues chamito-sémitiques :

Aire $2:$ « le mot des langues de l'aire seconde (les langues sémitiques) est un mot qui, par suite [d'une] division phonématique, siège pour moitié dans la langue, avec la racine pluri-consonantique, qui dit la substance-matière (p. ex. arabe k-tb, notion diffluente d' ' écrire») pour moitié dans le discours, avec les voyelles interpolées, qui disent la substance-forme (kataba, katib, kitab)» (ibid.)

Enfin, dans l'aire 3, le mot est une construction intégrale de langue (sens + forme) et l'unité de discours est la phrase. Cette aire correspond aux langues indo-européennes.

Ces « aires » doivent être conçues comme des états structuraux d'une pensée qui cherche en quelque sorte à inventer un langage ${ }^{3}$. Au cours de ce processus d'invention, la pensée passe, nécessairement, par un premier stade, l'aire 1, puis un second, l'aire 2, puis un troisième, l'aire 3 :

3 Cette démarche ne semble pas sans rapport avec certaines «expériences de pensée» en philosophie. 
La genèse du langage - on ne doit pas le perdre de vue - est expérimentale. On a exprimé comme on pouvait, manquant de moyens, et, empiriquement, on a inventé les moyens dont il était besoin, inventé et institué ${ }^{4}$ \{Note : En marge: Notons bien qu'il y a institution.\}. C'est l'institution de ces moyens, le fait de leur conférer une existence durable, non éphémère dans l'esprit, grâce à laquelle ils se présenteront à tout moment disponibles, qui a fait la langue. [...] (Guillaume 1957, p. 31)

Cette dynamique d'une expérimentation et d'une institution de certaines des solutions découvertes au cours de cette expérimentation se formule également, chez Guillaume, à partir des notions d'expression et de représentation que nous avons précédemment introduites. Ainsi, dans le cas de l'aire 1 :

On a commencé par des actes d'expression complexes de dicibilité mentale, orale, picturale, gestuelle, devenus par institution, et beaucoup de $<$ facilité $>$ dans l'institution, des actes de représentation. On eut ainsi la phrase deux fois : sous l'état éphémère d'un acte d'expression et sous l'état non éphémère, durable, d'actes de représentation. Ce fut l'époque du mot-phrase, long ou bref. Les éléments formateurs, issus de la mutation de l'expérience indicible nous le savons - en représentation dicible - ce avant quoi il n'y a pas de langage humain -, tombent sous le coup de la saisie phrastique qui les assemble et les agglutine, les soude en tant que première saisie formelle qu'aucune autre n'a précédée [...] (Guillaume 1957, p. 31-32)

Mais ce processus décrit sous la forme de la théorie des aires ne consiste pas seulement en une sédimentation de solutions expérimentées, d'une manière plus ou moins aléatoire, dans les pratiques langagières. La particularité de l'approche de Guillaume réside en effet dans l'idée qu'il existe une dynamique structurale interne à chaque aire, qui conduit vers l'aire suivante ${ }^{5}$ :

Je viens d'évoquer dans son entier l'histoire structurale du langage, ce qui me permet d'en revenir au sujet particulier de ma dernière leçon : l'étude de l'aire A' du langage et du mot primaire retenu - ce qui n'est pas voué à durer - dans cette aire $\mathrm{A}^{\prime} . \mathrm{La}<$ précarité> de cette rétention est le fait sur lequel j'appelle l'attention. Et les forces vives du langage auxquelles je m'intéresse dans l'examen que je fais de l'aire A' ne sont pas celles qui tendent à le maintenir dans cette aire, mais celles qui, par travail secret en ce sens, tendent à l'introduire à l'au-delà ontogénique de cette aire, l'aire A", et, par nouvel au-delà, à l'aire A"'. (Guillaume 1957, p. 41)

Il reste donc à expliquer d'où provient cette dynamique structurale. On atteint là le point le plus abstrait de la réflexion de Guillaume, qui en est aussi le fondement, et qui conduit à poser le concept de représentation comme condition structurale de l'existence du langage : 
Les conditions de grandeur et de forme du langage ne lui sont pas étroitement particulières. D'autres êtres que lui relèvent des mêmes conditions. Ce sont tous les êtres dont l'existence a la grandeur d'une infinitude et la forme congruente. Je cite au nombre de ces êtres, dans l'ordre de l'intégrant à l'intégré : l'univers, l'univers-espace, l'univers-temps, le langage. Tous les quatre sont des êtres d'infinitude, dont une caractéristique commune est de refuser l'extériorité. L'univers n'a pas d'extériorité : on ne rencontre rien qui ait existence en dehors de lui. On en peut dire autant de l'univers-espace : il a pour attribut l'infinitude et la non-extériorité qu'elle entraîne. On en peut dire autant aussi de l'universtemps : où a-t-il commencé ? où finira-t-il ? Pas d'extériorité. La poursuite d'un en-dehors est vaine. Il faut l'abandonner, revenir à la position de départ, par mouvement contraire. Le mouvement, en son infinitude, ne s'arrête pas. L'écoulement sans fin du temps, attesté par intuition, en témoigne.

Le langage est lui aussi un être d'infinitude, en ce sens qu'on n'en peut même pas poser le commencement dans le temps non plus que la fin, entre lesquels, dans le sens commencement $X$ fin, s'accroît indéfiniment la distance. La grandeur du langage, c'est cette distance inscrite entre une primitivité inaccessible, par remontée du temps, et une non-primitivité inaccessible, par descente du temps [...] Pas d'extériorité, une intériorité seulement. Or une intériorité sans extériorité constitue une amorphie ne satisfaisant pas aux exigences de la représentation. Les exigences de la représentation sont la binarité extériorité/intériorité. Ces exigences sont satisfaites d'emblée par les êtres spatiaux qui inscrivent leur finitude entre deux limites en dehors desquelles s'étend l'extériorité infinie [...] Promouvoir le langage à l'existence, c'est le promouvoir à la représentation - ce sans quoi rien n'existe pour l'esprit - et à cet effet, dans le cadre de l'intériorité seule existante d'un être d'infinitude, de rétablir la relation intériorité/extériorité. L'esprit humain y réussit par un artifice qui consiste à inscrire, dans l'infinitude de l'intériorité, une intériorité d'intériorité de grandeur finie et sténonome, dont l'intériorité de grandeur infinie fait l'extériorité. D'où la relation intériorité infinie/intériorité finie d'intériorité. En figure explicative :

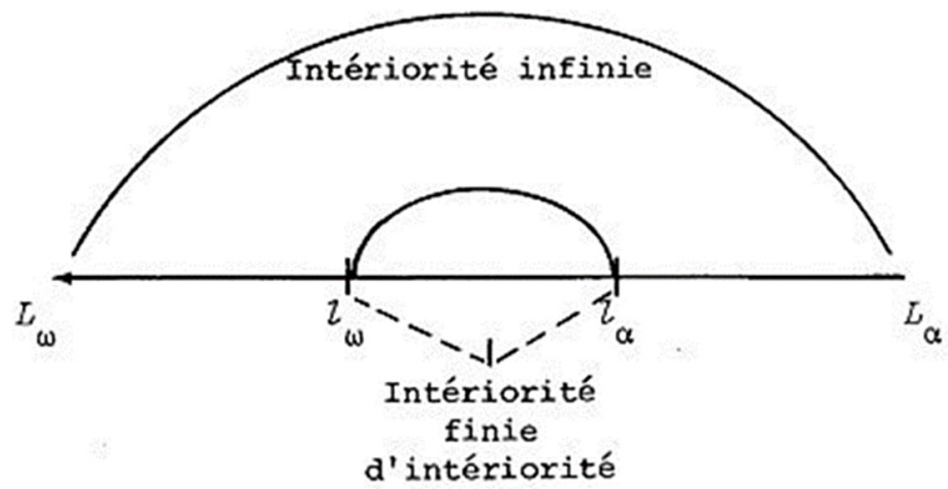

\footnotetext{
$L_{\alpha} Z_{\alpha}$ : extériorité première de l'intériorité finie d'intériorité.

$l_{\omega} L_{\omega}$ : extériorité seconde de l'intériorité finie d'intériorité.
} 
Le résultat est une distribution de l'entier du langage en trois aires : $L_{\alpha}-l_{\alpha} / l_{\alpha}$ $-l_{\omega} / l_{\omega}-L_{\omega}$. (Guillaume 1957, p. 13)

On notera en premier lieu que Guillaume appréhende l'être du langage non pas comme un type d'être particulier - un être de nature sémiotique - mais comme un être comparable à d'autres, et ici, spécifiquement, à d'autres êtres infinis : l'univers, l'espace, le temps. D'autre part, toute l'argumentation de Guillaume est fondée sur une proposition considérée comme nécessairement vraie, parce qu'il s'agit d'une vérité analytique: l'infini n'a pas d'extériorité ${ }^{6}$. Le langage étant un être d'infinitude (il s'agit d'un axiome ou d'un postulat), il n'a donc pas d'extériorité. Mais, troisième point, Guillaume pose une condition de possibilité de toute représentation: la représentation exige une binarité intériorité / extériorité. Cette idée, de nouveau présentée comme un postulat - mais qui n'est plus une vérité de type analytique - peut être interprétée ou justifiée en termes gestaltistes: toute représentation exige un contraste, une forme se détachant sur un fond. Les êtres spatiaux présentant de manière évidente cette binarité, ils peuvent être considérés comme prototypiques de la notion de représentation. Or, dernier point du raisonnement, Guillaume pose également une condition relative à «ce qui existe pour l'esprit», autrement dit une condition de l'existence des processus cognitifs : rien n'existe pour l'esprit sans représentation. Guillaume adopte donc très explicitement un point de vue représentationaliste : le langage ne peut exister qu'en tant qu'il est passible de représentation.

Le raisonnement que développe Guillaume dans cette Leçon du 6 décembre 1956, où il tente de répondre à la question «qu'est-ce que le langage?» en accordant une priorité à ce qu'il nomme la «définition ontogénique du langage » ${ }^{7}$, vise en premier lieu à justifier la théorie des trois aires. Mais, dans le cadre du présent propos, il m'intéresse surtout parce qu'il donne un nouvel éclairage sur la conception guillaumienne de la représentation. D'une part, comme on l'a souligné, il révèle la perspective représentationaliste de Guillaume. Mais il fait également apparaître que la linguistique de Guillaume est fondée sur des principes qui ne sont pas strictement linguistiques, ni cognitifs d'ailleurs, principes qu'il considère

6 Puisque l'infini peut être défini comme ce «qui est sans bornes, illimité (TLFi), l'absence d'extériorité de l'infini, reformulation de l'idée d'absence de bornes, peut être considérée comme faisant partie de la définition de l'infini.

7 Par opposition à une définition praxéogénique du langage, «celle des conditions auxquelles il doit satisfaire pour remplir le service - l'office - qu'on attend de lui » (ibid.). Le point de vue ontogénétique consiste, lui, à s'interroger sur «les conditions de forme et de grandeur auxquelles pour exister [le langage] doit satisfaire ainsi que toute chose vouée à l'existence » (ibid.). 
comme des « exigences absolues » ${ }^{8}$ ou des « inévitables » ${ }^{9}$ et qui sont ou bien des vérités analytiques, ou bien des sortes d'axiomes dont nous avons vu plusieurs exemples: «le langage est un être d'infinitude», "la représentation exige une binarité intériorité / extériorité », «promouvoir le langage à l'existence, c'est le promouvoir à la représentation», «sans la représentation rien n'existe pour l'esprit». Ces exigences absolues sont donc les axiomes sur lesquels Guillaume fonde (d'une manière très largement rétrospective, bien sûr) toute son analyse. Ce qui signifie que les «faits » linguistiques ne sont aucunement le point de départ de son raisonnement :

Là où l'enseignement n'est pas théorisant à partir d'intuitions fondamentales, l'enseignement peut consister à présenter un fait et une explication, puis le même fait ou d'autres avec d'autres explications. On confronte, on s'instruit, on n'explique pas vraiment. Cet enseignement est courant. Il n'est pas guillaumien. Ma tâche est de suivre, à partir de l'inévitable intuitionnel en position de postulat, un chemin qui me mène à la rencontre des faits. (Guillaume 1957, p. 89-90)

L'observation des «faits » empiriques conduit au mieux à des généralisations insuffisantes (car plusieurs types de généralisations sont possibles pour les mêmes faits), et à des descriptions incomplètes car limitées au plan du discours. Une explication non réduite à la généralisation suppose un seuil au-delà duquel on quitte la simple généralisation pour l'élaboration ou l'imagination d'un modèle théorique qui devra ensuite être confronté aux «faits» empiriques. Si j'ai pris la peine de développer ce point ici, c'est parce qu'il me semble que cette caractéristique de l'épistémologie guillaumienne peut difficilement être rapportée à une filiation saussurienne. Peut-être même est-elle radicalement étrangère à la perspective de Saussure.

Mais revenons à la question de la représentation. Trois des quatre axiomes que nous avons relevés la concernent directement. Elle apparaît ainsi comme une condition de tout processus cognitif («sans la représentation rien n'existe pour l'esprit») et par conséquent comme une condition de possibilité du langage

8 «La théorie, superlatif du comprendre, doit pour m'agréer satisfaire aux conditions formelles suivantes : aller à la rencontre des faits en position antagoniste, certes, mais prendre son départ non pas au fait mais à une exigence absolue, inévitable, et cheminer d'exigences absolues en exigences absolues jusqu'à la rencontre des faits » (Guillaume 1957, p. 85)

9 «Et enfin, l'idée majeure, souveraine, <est> qu'il ne faut entrer en science du langage qu'après avoir suscité en soi par la méditation suivie, - et "rangeant" au plus près, comme on dit en langage de marin, l'inévitable en pensée - un rêve constructif honnête, probe en ses raisons, de ce qu'est en sa nature, qui est le profondément caché de lui-même, le langage humain, langage situé dans l'homme pensant <et $>$ réfractant, réfléchissant, diffractant la lucidité humaine, tout de même que le langage situé en mon chien réfracte, réfléchit, diffracte la lucidité canine.» (Guillaume 1959, p. 175) 
lui-même («promouvoir le langage à l'existence, c'est le promouvoir à la représentation »). En outre, Guillaume pose une condition de formation de toute représentation : «la représentation exige une binarité intériorité / extériorité ». On remarquera enfin que ce dernier axiome est le plus spécifique et que c'est sur lui qu'est essentiellement fondée la théorie des aires: d'une manière générale, ces axiomes guillaumiens se vérifient par leur pertinence explicative. Puisqu'ils sont présentés comme des axiomes, ils ne sont donc pas soumis à l'épreuve des faits; mais il demeure que lorsqu'ils sont suffisamment spécifiques, précis, rien n'empêche de les soumettre à une vérification expérimentale.

Au total, si on si l'on dresse le bilan des fonctions de la représentation chez Guillaume, quatre types d'utilisations de ce concept peuvent être distinguées :

1. La représentation comme actualisation (transition langue $>$ discours, puissance $>$ effet).

2. La représentation comme principe sémiologique: le signifiant représente le signifié.

3. La représentation comme système (p. ex. le système verbo-temporel du français comme représentation du temps).

4. La représentation et les "problèmes de pensée»: les êtres parlants trouvent des solutions linguistiques à des problèmes de représentation (p. ex. l'émergence de l'article en français).

5. La représentation comme mouvement de l'histoire structurale des systèmes de représentation que sont les langues (théorie des aires).

On remarquera que, chez Guillaume, la relation de représentation entre signifiant et signifié est asymétrique : le signifiant représente le signifié, mais le signifié ne représente pas le signifiant. Il s'agit là encore d'une différence avec Saussure: puisque le rapport entre signifiant et signifié est de la nature d'une dualité, il n'y a pas d'asymétrie «cognitive» entre signifiant et signifié dans la perspective saussurienne, donc pas de représentation. Cette relation de représentation entre signifiant est signifié prend place dans un système explicatif fondé en outre sur deux autres types de relations: l'actualisation et le conditionnement structurel (fig. 5).

Les signifiants puissanciels représentent des signifiés d'effet. Ces signifiants puissanciels produisent des signifiants d'effet (les représentations phonologiques sont antérieures et sont actualisées par des signifiants d'effet). Les signifiés d'effet sont des actualisations des systèmes (qui sont les signifiés de puissance) et les signifiés de puissance proviennent d'une «mécanique intuitionnelle», expression couramment utilisée par Guillaume et qui vise à caractériser un ensemble de contraintes cognitives (p. ex. l'opposition intériorité / extériorité), qui elles-mêmes conditionnent les structures des systèmes linguistiques. 


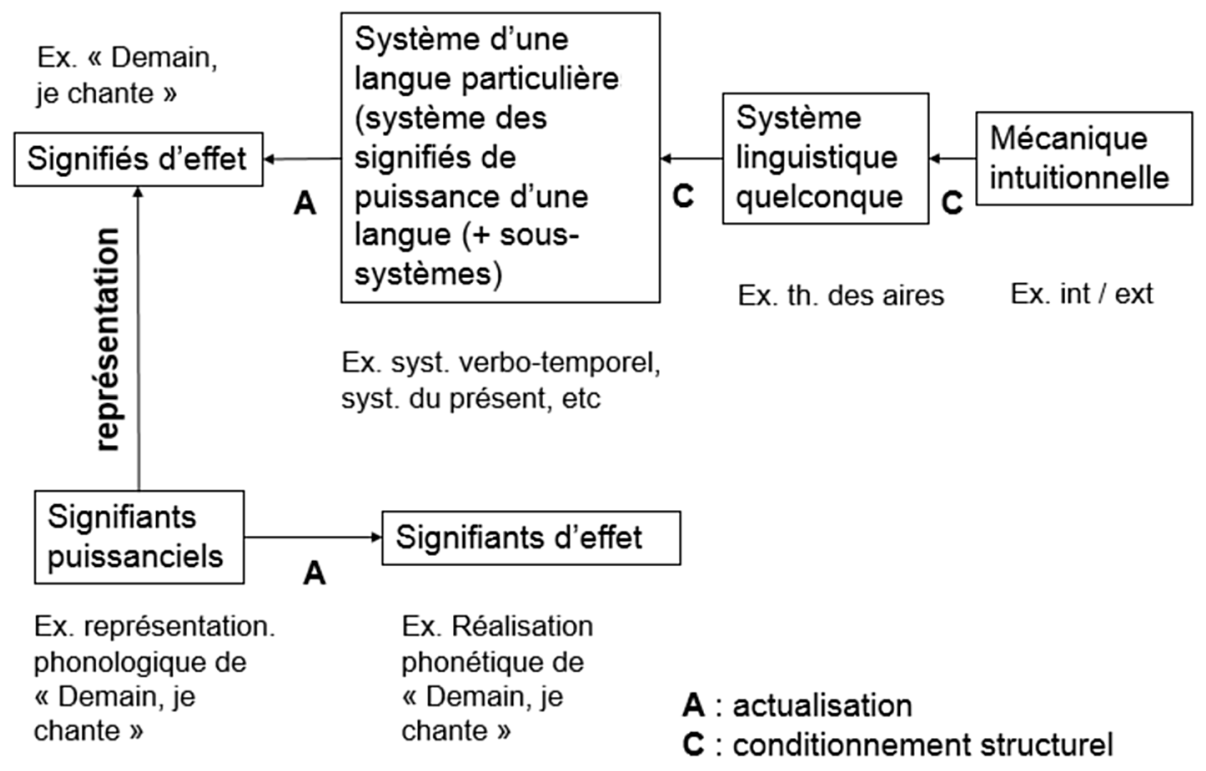

Figure 5 : Représentation, actualisation et conditionnement structurel chez G. Guillaume.

\section{LA QUESTION DU SYSTÈME DE LA LANGUE}

Pour comprendre le déplacement qu'effectue Guillaume par rapport à Saussure, il convient revenir plus précisément à la notion de système. Car lorsque Guillaume cite Saussure, c'est le plus souvent pour se définir lui-même comme un successeur de Saussure au sujet de la question des systèmes linguistiques. Autrement dit, Guillaume se veut saussurien quand il envisage la langue comme système:

Un large courant d'approbation a accueilli en 1916, quand parut le Cours de linguistique générale de Ferdinand de Saussure, l'opinion qu'y professe l'auteur que la langue est un système et doit être étudiée en elle-même comme telle; et ce n'est que plus tard, récemment, que des réserves avancées de plusieurs côtés quant à la justesse de ce jugement du grand linguiste ont tardivement ouvert un débat au cours duquel il est apparu que si c'est une vérité aperçue de la plupart que la langue est un système, - qu'elle n'est pas sous les signes qui lui prêtent un corps, une simple individuation d'idées, mais conjointe à cette individuation, une représentation des moyens formels mécaniquement liés, faisant un tout mécanique cohérent, que l'esprit emploie à une saisie, qu'il voudrait intégrale [...], du pensable, - la preuve que cette vérité requiert (la science ne vit pas de vérités, elle vit de preuves, disait Meillet) n'avait pas été produite. Le système ne fût-ce que celui d'une langue - n'avait point été démonté : on le déclarait existant sans en faire voir distinctement les rouages et encore moins la loi d'assemblage qui, faute d'exister autrement que comme relativité réciproque des composants, ne peut être perçue que dans l'abstrait par les yeux de l'esprit. Lecteur dès son apparition du Cours de linguistique générale de Saussure, il nous 
a semblé, dès ce moment, et toujours depuis, que la tâche de la preuve et de l'explication complète en la matière était un legs du maitre à ses disciples. Ce sentiment, durant plus de trente ans, a inspiré l'ensemble de nos recherches et aujourd'hui encore il inspire cette étude».

Or Guillaume pense le système de la langue dans le sens de la «systématicité », de l'organisation interne d'un système. Et, dans le Cours de linguistique générale, le «système» est bien envisagé dans cette perspective:

La langue est un système qui ne connaît que son ordre propre. (Saussure 1975, p. 43)

Une langue constitue un système. Si, comme nous le verrons, c'est le côté par lequel elle n'est pas complètement arbitraire et où il règne une raison relative, c'est aussi le point où apparaît l'incompétence de la masse à le transformer. Car ce système est un mécanisme complexe; l'on ne peut le saisir que par la réflexion; ceux-là mêmes qui en font un usage journalier l'ignorent profondément. (ibid., p. 107)

Tout ce qui a trait à la langue en tant que système demande, c'est notre conviction, à être abordé de ce point de vue, qui ne retient guère l'attention des linguistes : la limitation de l'arbitraire. C'est la meilleure base possible. En effet, tout le système de la langue repose sur le principe irrationnel de l'arbitraire du signe qui, appliqué sans restriction, aboutirait à la complication suprême; mais l'esprit réussit à introduire un principe d'ordre et de régularité dans certaines parties de la masse des signes, et c'est là le rôle du relativement motivé (ibid., p. 182)

Si le mécanisme de la langue était entièrement rationnel, on pourrait l'étudier en luimême ; mais comme il n'est qu'une correction partielle d'un système naturellement chaotique, on adopte le point de vue imposé par la nature même de la langue, en étudiant ce mécanisme comme une limitation de l'arbitraire.» (ibid., p. 183)

Mais, bien évidemment, la notion de système présente également chez Saussure un autre contenu, qui semble totalement négligé par Guillaume, la langue comme système au sens d'un système de valeurs :

Pour se rendre compte que la langue ne peut être qu'un système de valeurs pures, il suffit de considérer les deux éléments qui entrent en jeu dans son fonctionnement: les idées et les sons. (ibid., p. 155)

Pour les sciences travaillant sur des valeurs, cette distinction devient une nécessité pratique, et dans certains cas une nécessité absolue. Dans ce domaine on peut mettre les savants au défi d'organiser leurs recherches d'une façon rigoureuse sans tenir compte des deux axes, sans distinguer le système des valeurs considérées en soi, de ces mêmes valeurs considérées en fonction du temps. C'est au linguiste que cette distinction s'impose le plus impérieusement; car la langue est un système de pures valeurs que rien ne détermine en dehors de l'état momentané de ses termes. » (ibid., p. 115-116)

Quelle que soit la place de la langue parmi les autres systèmes sémiologiques, on l'aura fixée quand on aura déterminé qu'elle est un système de valeurs. Il faudra 
trouver sa base dans la collectivité ; c'est elle qui est créatrice de la valeur. Donc, $<1)>$ la valeur n'existant pas en dehors de la collectivité, on étudiera vainement la valeur dans ce qui se passe chez les individus $<$ isolés ; $>$ de même les variations ne seront pas non plus livrées à l'initiative des individus. Mais, $<2)><$ comme pour toute valeur dépendant de facteurs sociaux, $>$ ce n'est pas ce qui entre dans un signe linguistique qui peut donner une idée de ce qu'est ce signe. Tout cela n'est que la matière utilisée; la valeur peut varier sans que ces éléments varient. Nous en revenons à ce que nous disions: la langue n'est pas dans ce qui nous frappe anthropologiquement, dans ce qui est indispensable pour la produire (le son, l'idée, considérés seuls). Nous aurons certainement un objet très complexe mais pas plus complexe que toute autre valeur. Ce que nous avons dit suffira pour déterminer ce qui rentre ou qui ne rentre pas dans la linguistique et pour en classer les différents sujets. (Saussure 1997, p. 29, cité par Toutain 2012, p. 3048)

Il y a donc une ambiguïté de la notion de système chez Saussure, que Guillaume ignore en se concentrant sur la langue comme «ensemble où tout se tient» (Meillet, Guillaume). Guillaume a connu la pensée de Saussure par le Cours de linguistique générale mais aussi grâce à l'enseignement de Meillet, dont il rapporte souvent cette formule. Et, passant à côté de l'invention saussurienne du concept de valeur, l'idée essentielle demeure pour lui celle de la cohérence. Un système linguistique est un système parce qu'il est cohérent ${ }^{10}$. Or, ce principe de cohérence, caractéristique de la démarche guillaumienne, semble radicalement opposé à l'idée que Saussure se fait du système ou du «mécanisme » de la langue :

Si le mécanisme de la langue était entièrement rationnel, on pourrait l'étudier en lui-même; mais comme il n'est qu'une correction partielle d'un système naturellement chaotique, on adopte le point de vue imposé par la nature même de la langue, en étudiant ce mécanisme comme une limitation de l'arbitraire (Saussure 1975, p. 183).

Si les phénomènes relevant de la motivation relative et qui constituent la dimension de la langue comme «mécanisme », c'est-à-dire comme système au sens où l'entend Guillaume, sont envisageables selon Saussure uniquement comme des limitations de l'arbitraire du signe, il sont appréhendés par Guillaume comme des structurations primaires, non pas « secondaires » comme limitations d'une essence chaotique de la langue. Puisque les représentations produites par l'esprit humain sont, pour Guillaume, le résultat de processus dotés d'une cohérence propre, la langue n'est pas vue comme chaotique par essence mais comme régulière, avec des résidus chaotiques (l'arbitraire) issus de la «suffisance expressive » qui régit les signifiants, imparfaitement modelés sur les signifiés. Une note de l'article «Observation et explication et explication dans la science du langage» indique

10 La cohérence concerne uniquement, chez Guillaume, le plan des signifiés. Les signifiants sont régis par un principe plus souple, celui de la «suffisance expressive». 
clairement cette hypothèse d'un ordre, d'une cohérence à trouver dans le(s) système(s) de la langue :

Certains, erronément, jugeront que ces opérations, si mécaniquement cohérentes et symétriques soient-elles, sont trop compliquées pour avoir été et être la réalité linguistique. À la racine de ce jugement erroné, il y a l'idée, en soi étrange, que dans l'univers regardé par l'homme pensant, hors lui, l'univers où il habite et duquel il fait partie, s'accuserait un ordre inhérent, d'une nature singulière, que découvre lentement, apte à le comprendre, l'intelligence humaine; cependant que dans l'univers regardant, la langue, qui habite dans l'homme pensant, s'accuserait un désordre inhérent. Un confrère ne me disait-il pas récemment : «la langue n'est, au fond, qu'un tissu de calembours et de coq-à-l'âne». Combien plus raisonnable serait d'admettre que dans le mentalisme de la langue (suspendu à un physique qui n'est point lui) règne un ordre inhérent, d'une nature singulière lui aussi, que n'a pas su jusqu'à présent reconnaître la science du langage, mais qu'il n'est pas au-dessus de ses forces de déceler. (Guillaume 1964, p. 286)

Dans le «mentalisme de la langue », autrement dit dans sa dimension cognitive, où s'élaborent les représentations constitutives des systèmes, règne un «ordre inhérent». Cet ordre, on l'a vu, se formule chez Saussure sous la dénomination de «motivation relative» comme une limitation de l'arbitraire mais, dans la perspective opposée qu'est celle de Guillaume, c'est au contraire l'arbitraire qui apparaît comme une nécessité résiduelle et la motivation (ou l'analogie) comme le principe structurant fondamental.

La question de l'arbitraire du signe joue donc un rôle essentiel dans le clivage entre les perspectives des deux linguistes. Nous avons pu observer qu'il existe manifestement un rapport entre d'une part l'absence de thématisation du concept de représentation chez Saussure et la position centrale du principe de l'arbitraire, d'autre part le représentationnalisme de Guillaume et son insistance sur la cohérence des systèmes, qui minore radicalement ce qu'ils conservent d'arbitraire. Ces deux perspectives s'expliquent notamment par le fait que Guillaume est en premier lieu attentif à ce que les langues doivent à la dimension cognitive de l'humain tandis que Saussure accorde une importance de premier plan à la dimension collective de la langue, d'où proviennent la théorie de la valeur et le principe de l'arbitraire. Mais, comme nous l'avons vu, l'appréhension de la langue comme système au sens guillaumien, c'est-à-dire comme «mécanisme de la langue », est également présente chez Saussure. Par conséquent, considérer qu'il convient de penser la représentation à partir du concept d'iconicité (ou du concept d'analogie ${ }^{11}$ ), comme le font la plupart des linguistiques cognitives contemporaines, ne signifie pas pour autant qu'on adopte un point de vue anti-saussurien. Il demeure que la plupart des linguistes qui ont thématisé la question de l'iconicité se 
sont eux-mêmes définis ainsi ${ }^{12}$. Il s'agit donc d'un malentendu, qui trouve son origine dans l'ambiguïté de la définition saussurienne du système et que les spécialistes de l'iconicité linguistique gagneraient non pas à diffuser, plus ou moins volontairement, mais plutôt à éclaircir. La thématisation linguistique de l'analogie ou de l'iconicité est donc autant saussurienne que guillaumienne.

\section{BIBLIOGRAPHIE}

Boone, Annie et Joly, André, 1996. Dictionnaire terminologique de la systématique du langage, Paris, Harmattan.

Guillaume, Gustave, 1919. Le Problème de l'article et sa solution dans la langue française, Paris, Hachette. Réédition de 1975, Paris et Québec, A.-G. Nizet et Les Presses de l'Université Laval.

- 1929 [1993]. Temps et Verbe. Théorie des aspects, des modes et des temps, Paris, H. Champion.

- 1939 [1992]. Leçons de linguistique de Gustave Guillaume, 1938-1939, Leçons de l'année 1938-1939, Québec, Presses de l'Université Laval, et Lille, Presses universitaires de Lille.

- 1945 [1991]. Leçons de linguistique de Gustave Guillaume, 1944-1945, Québec, Presses de l'Université Laval, et Lille, Presses universitaires de Lille.

- 1949 [1974]. Leçons de linguistique de Gustave Guillaume, 1949-1950, Série A, «Structure sémiologique et structure psychique de la langue française (II)», 4.

- 1959 [1995]. Leçons de linguistique de Gustave Guillaume, 1958-1959 et 1959-1960, Québec, Presses de l'Université Laval, et Paris, Librairie C. Klincksieck, 1995.

- 1964. Langage et Science du Langage, Paris et Québec, A.-G. Nizet et Presses de l’Université Laval.

- 1957 [1982]. Leçons de linguistique de Gustave Guillaume, 1956-1957, «Systèmes linguistiques et successivité historique des systèmes II», Québec, Presses de l’Université Laval, et Lille, Presses Universitaires de Lille [1982, Québec, Les Presses de l'Université Laval et Paris, Klincksieck].

- 1986. Principes de linguistique théorique, Québec, Presses de l'Université Laval.

Haiman, John, 2006. "Iconicity», The Encyclopedia of Language and Linguistics, R.E. Asher (ed.), Oxford, Pergamon Press, vol 3, 1629-1633.

Monneret, Philippe, 2003. Notions de neurolinguistique théorique, Éditions Universitaires de Dijon.

- 2014. "L'iconicité comme problème analogique», Le Français Moderne, 1, 46-77.

Saussure, Ferdinand de, 1975. Cours de linguistique générale, Payot.

- 2002. Écrits de linguistique générale, Paris, Gallimard.

- 1997. Deuxième cours de linguistique générale (1908-1909), d'après les cahiers d'Albert Riedlinger et Charles Patois, édition d'Eisuke Komatsu et George Wolf, Amsterdam, Elsevier.

Toutain, Anna-Gaëlle, 2012. Montrer au linguiste ce qu'il fait. Une analyse épistémologique du structuralisme européen (Hjelmslev, Jakobson, Martinet, Benveniste) dans sa filiation saussurienne, Thèse de doctorat de l'Université Paris-Sorbonne. [https://tel.archives-ouvertes.fr/tel-00788676]

12 À titre d'exemple, la définition donnée par John Haiman de l'iconicité dans The Encyclopedia of Language and Linguistics présente l'iconicité comme un affranchissement à l'égard du « dogme » de l'arbitraire : «The first significant challenge to this dogma [1'arbitraire du signe] in the respectable philological tradition is Roman Jakobson's famous article "Quest for the essence of language" (1965) [...].» (Haiman 2006, p. 1269). 